25 fractions of 2.0 Gy (total 50.0 Gy), 13 fractions of 3.3 Gy (total $42.9 \mathrm{~Gy}$ ), or 13 fractions of 3.0 Gy (total 39.0 Gy) over a 5-week period. After a median follow-up of 9.7 years, 965 $(68 \%)$ of all patients were alive. Local relapse, distant relapse, and second primary breast cancer were reported in $3 \%, 3 \%$, and $2 \%$ of all surviving patients, respectively. The risk of local recurrence after 10 years was $12.1 \%$ in the 50 Gy group, $9.6 \%$ in the 42.9 Gy group, and 14.8 in the 39 Gy group. The difference in risk of local recurrence between the 42.9 and 39 Gy schedules was statistically significant $(P=0.027)$. Breast cancer sensitivity to fraction size was estimated to be 4.0 Gy, which is comparable to the sensitivity of healthy tissue (3.0-5.0 Gy).

If the results of this analysis are substantiated by other trials, radiotherapy regimens for patients with breast cancer could be made simpler, more convenient and more cost-effective.

Original article Owen JR et al. (2006) Effect of radiotherapy fraction size on tumour control in patients with early-stage breast cancer after local tumour excision: long-term results of a randomised trial. Lancet Oncol 7: 467-471

\section{Longer-term estrogen therapy is associated with increased risk of breast cancer}

Researchers in the US have investigated whether long-term unopposed estrogen therapy is associated with an increased risk of invasive breast cancer. The Nurses' Health Study included women who became menopausal and underwent a hysterectomy before or during the period 1980-2002 (28,835 women as of June 2002).

During the study period 934 women were diagnosed with invasive breast cancer, 226 of whom had never received estrogen therapy and 708 of whom were current users of estrogen therapy (351 breast cancer cases in patients who had used estrogen therapy in the past were not discussed in this paper). In current estrogen therapy users, a linear increase in breast cancer risk was seen with increasing duration of use $\left(P_{\text {trend }}<0.001\right)$, but the risk did not become statistically significant until estrogen therapy had been used for $\geq 20$ years (relative risk 1.42, 95\% $\mathrm{Cl} 1.13-1.77)$. The association of breast cancer with longer-term estrogen therapy was stronger when only tumors positive for both estrogen and progesterone receptors were considered, and the association was mainly seen in women with BMI of less than $25 \mathrm{~kg} / \mathrm{m}^{2}$.

The authors conclude that estrogen therapy was associated with increased risk of breast cancer only after longer-term use ( $\geq 20$ years). They suggest that alternative treatment options should, therefore, be explored for women who require longer-term estrogen therapy, such as those with osteoporosis.

Original article Chen WY et al. (2006) Unopposed estrogen therapy and the risk of invasive breast cancer. Arch Intern Med 166: 1027-1032

\section{Response to adjuvant chemotherapy is affected by HER2 amplification}

The National Cancer Institute of Canada Clinical Trials Group Mammary. 5 trial randomized premenopausal women with node-positive breast cancer to receive six cycles of either CEF (cyclophosphamide, epirubicin and fluorouracil) or CMF (cyclophosphamide, methotrexate and fluorouracil) chemotherapy. Relapse-free and overall survival rates were greater in the CEF group, but CEF was associated with higher adverse event rates.

In a follow-up trial, researchers in Canada have evaluated HER2 amplification and overexpression (by immunohistochemical analysis, polymerase chain reaction and fluorescence in situ hybridization) in 639 formalin-embedded specimens from 710 women in the Mammary. 5 cohort, to investigate whether HER2 amplification or overexpression is associated with an improved response to anthracycline-containing chemotherapy regimens such as CEF.

Women with HER2 amplification were at significantly greater risk of relapse $(P=0.03)$ and death $(P<0.001)$ than women whose tumors did not show HER2 amplification. Tumors with HER2 amplification showed a better response to CEF than CMF in terms of relapse-free survival $(P=0.003)$ and overall survival $(P=0.06)$, but the two regimens resulted in similar survival rates in tumors without HER2 amplification. Overexpression of HER2 protein was also associated with improved response to CEF in terms of relapse-free survival.

The authors conclude that the increased benefit of adjuvant CEF in women with nodepositive breast cancer is mainly a result of the improved response in women with tumors 